\title{
Emptying a Paradox of Ground
}

\author{
Jack Woods ${ }^{1}$
}

Received: 21 June 2016 / Accepted: 4 April 2017 / Published online: 10 July 2017

(C) The Author(s) 2017. This article is an open access publication

\begin{abstract}
Sometimes a fact can play a role in a grounding explanation, but the particular content of that fact make no difference to the explanation-any fact would do in its place. I call these facts vacuous grounds. I show that applying the distinction between-vacuous grounds allows us to give a principled solution to Kit Fine and Stephen Kramer's paradox of (reflexive) ground. This paradox shows that on minimal assumptions about grounding and minimal assumptions about logic, we can show that grounding is reflexive, contra the intuitive character of grounds. I argue that we should never have accepted that grounding is irreflexive in the first place; the intuitions that support the irreflexive intuition plausibly only require that grounding be non-vacuously irreflexive. Fine and Kramer's paradox relies, essentially, on a case of vacuous grounding and is thus no problem for this account.
\end{abstract}

Keywords Grounding · Paradoxes of ground · Irreflexivity

Interest in the putatively explanatory relation of metaphysical grounding and, in particular, its "logic" has recently been the focus of much attention. ${ }^{1}$ Grounding has often been taken to be a strict partial order-i.e. to be an irreflexive, asymmetric, transitive relation. However, there have been important challenges to the claim that

\footnotetext{
${ }^{1}$ See [24] for a recent survey, [7, 26, 27] for the kick-off of the contemporary research program, and [23, $26]$ for a defense of the coherence of the notion and that it is an explanatory relation.
}

Jack Woods

j.e.woods@gmail.com

1 University of Leeds, Leeds, West Yorkshire, UK 
grounding is irreflexive in the sense that a fact never occurs simultaneously as a grounded fact and one of its grounds: ${ }^{2}$

$$
\text { IRREFLEXIVITY: }\lfloor\ldots, A \nless A\rfloor
$$

While IRREFLEXIVITY is a natural assumption, especially given the use of metaphysical grounding in explicating notions of dependence, metaphysical explanation, and suchlike, it has recently been challenged. The most interesting challenges have come from Fine [8] and, developing a simplified version of Fine's point, Krämer [13]. ${ }^{3}$ They show that other natural assumptions about grounding, especially assumptions involving the grounding of existential facts, imply that there are cases of reflexive ground.

In light of the challenge, Krämer suggests (but does not ultimately endorse) restricting the existential grounding principle that true existential claims are grounded in their true instances and Fine mentions the same before developing other costly options such as the highly inegalitarian (and rather puzzling) thought that only a particular instance grounds an existential fact. In my view, we can get off cheaper than this; there is a natural restriction of irreflexivity which preserves much, and perhaps all, of the intuition driving the thought that grounding is irreflexive while accommodating Fine and Krämer's examples. ${ }^{4}$

My purpose here is to develop this solution. I do so explicitly in the service of offering an alternative to the orthodox irreflexive picture of grounding, albeit one which I think particularly natural and useful. ${ }^{5}$ While I will provide reasons below to favor my approach, I will not give conclusive arguments in its favor. By my lights, this is the most natural solution to the puzzles Fine and Krämer have developed, but I privilege aspects of the grounding relation-such as its explanatory aspects - that others may think of relatively minor importance. When, as in the case of grounding, intuitions differ so widely about its nature, it is useful to have a slate of options for accommodating various intuitions. The option I develop here picks up on a hitherto unnoticed aspect of certain grounding relations-sometimes, the grounds of some

\footnotetext{
${ }^{2}$ I represent a total grounding relation by $\lfloor\ldots<B\rfloor$ where $B$ is the grounded fact, the constituents to the left of it the grounds of $B$. Later on, I will use $\lfloor\ldots \prec B\rfloor$ for the relation of partial grounding where some facts partially ground $B$ if they are part of a set of total grounds for that fact. '...', in the inset, indicates that there may be additional facts besides $A$ among the grounds on the left of ' $<$ '. The floor brackets are just to enhance readability.

${ }^{3}$ I am ignoring here other challenges arising in the context of ontological dependence and related notions like metaphysical causation, such as those of Barnes [2], Bliss [3], Jenkins [11], and Paseau [17]. These challenges strike me as less immediate and pressing than the sort of very general challenge raised by Krämer and Fine and potentially admit of independent solution.

${ }^{4}$ Obviously, if there are cases of reflexive grounding, then these very cases are also recherché cases of symmetric ground. So ASYMMETRY will have to be weakened to ANTISYMMETRY.

${ }^{5}$ See Fine's sensible remarks on treating the development of a logic of ground in terms of reflective equilibrium [9] (p. 97). While I differ on the slate of available options, I agree with Fine that there is no solution that comes without some cost. The resolution I provide below strikes me as the least costly, but, as always, de gustibus.
} 
instance of grounding are vacuous in the sense that their particular content does no substantive work in grounding the grounded fact. Any fact with any content would do just as well in grounding the grounded fact (in a precise sense I spell out below). ${ }^{6}$

In the cases of reflexive grounding Fine and Krämer identify, the grounded fact would be grounded by any fact at all, including itself. This means that the identity of the ground and the fact it grounds is not essential to the work done by the ground in grounding the grounded fact. If we can reconcile ourselves to admitting that in these special kinds of cases-cases of vacuous grounding - grounding can be reflexive, then we can save most of the intuition that grounding is irreflexive without abandoning the exceedingly plausible claim that existential facts are grounded in their true instances. Grounding is not irreflexive, but it is substantively irreflexive-it is never the case that something is non-vacuously grounded in itself.

Fine and Krämer's challenges are closely related and Krämer's is the simplest to state, so we will work with it in what follows. ${ }^{7}$ I concentrate on the notion of total ground at first even though the puzzle arises for both total and partial grounding as both Fine and Krämer note. Partial grounding will be discussed after I discuss the puzzle for total ground (objection 5). My solution extends naturally to solve reflexive cases of partial grounding so long as a partial ground is understood as being part of some set of total grounds. Although the target notion is grounding, understood as a notion of metaphysical explanation occurring between facts, Fine and Krämer's puzzles may arise for other irreflexive and transitive explanatory relations in the vicinity of grounding. As far as I can see, for those on which it clearly arises, my solution applies. $^{8}$

I assume that grounding is best expressed as a relation between facts-which I take to be true structured propositions-not as an operator on sentences, though

\footnotetext{
${ }^{6}$ Donaldson [6] raises a related puzzle of property grounding involving a grounding principle for $\lambda$ abstraction and grounding principles for second-order quantifiers. Donaldson shows that these principles yield $\lfloor\langle\lambda . x \exists X X x\rangle a<\langle\lambda . x \exists X X x\rangle a\rfloor$, violating IRREFLEXIVITY in the presence of $\lambda$-abstraction and second-order quantification. This puzzle came to my attention too late to address it here though I will note two things. First, we need to first account for when $\langle\lambda \cdot x \varphi(x)\rangle(t)$ and $\varphi(t)$ are distinct propositions before we can address how plausible the $\lambda$-abstraction principles generating the puzzle are. If, for example, we treat $\langle\lambda . x \exists X X x\rangle a$ and $\exists X X a$ as expressing the same proposition, as well we might, then the puzzle doesn't get off the ground. Second, $\langle\lambda . x \exists X X x\rangle$ occurs vacuously in $\lfloor\langle\lambda . x \exists X X x\rangle a<\langle\lambda . x \exists X X x\rangle a\rfloor$ in the sense defined in [33]. This opens up room to use my solution below, suitably adapted, to explain away these cases of reflexive ground. I hope to address these cases elsewhere.

${ }^{7}$ As Krämer notes, the central difference between his and Fine's account has to do with operating in a language containing propositional quantification and one without. Nothing significant I say below turns on which way we formulate the puzzle, so I shall not worry about the difference.

${ }^{8}$ Many other relations which have been lumped with grounding are ones on which the puzzle doesn't apply. As noted by a referee, for instance, causation appears irreflexive and transitive, but the analogue of EXISTENTIAL GROUNDING seems obviously implausible for it; instances do not plausibly cause their generalizations. Metaphysical causation and ontological dependence also seem to be irreflexive and transitive, but the exact relationship of these to logical grounding principles like EXISTENTIAL GROUNDING and to metaphysical explanation is itself not obvious [12]. Consider, in contrast, a relation of conceptual priority, like that plausibly obtaining between $p$ and $p$ is true. This likewise seems irreflexive and transitive, and principles like EXISTENTIAL GROUNDING seems plausible for it. If it is distinct from grounding, then the sort of solution I moot here seems applicable to it as well.
} 
little turns on which is chosen between these two options. ${ }^{9}$ Treating propositions as structured and facts as true structured propositions allows a useful and elegant treatment of the combination of propositional quantification and grounding, but it is not absolutely essentially to the broad structural point I am making. Notationally, since I am assuming that propositions are structured sentence-like entities, I shall use sentences enclosed in angle brackets-like $\langle\ldots\rangle$-when context doesn't immediately disambiguate. Finally, I presume that propositions stand in entailment relations of the requisite sort.

Let us turn to setting up Fine and Krämer's challenge. Assume we are working with a background logical theory containing non-substitutional propositional quantification. That is, given a structured proposition $\varphi(A)$, containing the proposition $A$ in "sentential" position, we can existentially generalize to obtain the proposition $\exists p \varphi(p)$, where $p$ is a variable ranging over propositions and $A$ is free for $p$ in $\varphi(A) .{ }^{10}$ Similarly for universal elimination. So, for example, $\varphi(A)$ entails the proposition $\exists p \varphi(p)$ and $\forall p \varphi(p)$ entails $\varphi(A)$ (see $[19,32]$ for further details. $)^{11}$

More concretely, $\langle\exists p(p \wedge$ some logicians do not like use and mention $)\rangle$ is entailed by 〈some metaphysicians like use and mention and some logicians do not $\rangle$ since 〈some metaphysicians like use and mention〉 occurs in sentential position in 〈some metaphysicians like use and mention and some logicians do not $\rangle$. Similarly, $\exists p p$ is entailed by 〈some metaphysicians like use and mention〉 as 〈some metaphysicians like use and mention〉 occurs in sentential position in 〈some metaphysicians like use and mention $\rangle$.

On reasonable (non-substitutional) treatments of propositional quantification, $\exists p p$ is a theorem:

$p f$. Suppose $\neg \exists p p$. Then $\exists p p$, since $\exists p p$ is entailed by $\neg \exists p p$ (as $\neg \exists p p$ is free for $p$ in $\neg \exists p p$ ). Tilt. So, $\exists p p$.

Now, given the standard claim that true propositions whose dominant logical component is an existential quantifier are grounded in their true instances-EXISTENTIAL GROUNDING-we have:

$$
\lfloor\varphi(A)<\exists p \varphi(p)\rfloor
$$

for $\varphi(A)$ a fact containing $A$ and $A$ free for $p$ in $\varphi(A)$. That is, we have that $\varphi(A)$ fully grounds $\exists p \varphi(p)$. Since $\exists p p$ is free for $p$ in $\exists p p$, we have the particular case:

$$
\lfloor\exists p p<\exists p p\rfloor
$$

\footnotetext{
${ }^{9}$ I make no heavy duty claims about what structured propositions are; I just assume for every sentence that there is a proposition corresponding to it, that propositions have contents, in some intuitive sense, and that facts are true propositions. Other conventions are possible, though my solution could be adapted, mutatis mutandis, to these, so long as there is sufficient sentence-like structure in the objects which are taken to flank the grounding relation (or, with some complication of the examples, in the objects that a grounding operator applies to.)

${ }^{10}$ Roughly, $A$ is free for $p$ in $\varphi(A)$ just in case $A$ does not fall inside of the scope of a variable-binding constituent which binds $p$. For example, if our proposition were $\langle\exists p$ ( $p \wedge$ grass is not green $)\rangle$, then $\langle$ grass is green $\rangle$ is not free for $p$ in $\langle\exists p(p \wedge$ grass is not green $)\rangle$.

${ }^{11}$ This fairly minimal construal of propositional quantification, involving only quantifying into sentential position, is all we need to run Fine and Krämer's challenge. My solution to their puzzle extends naturally to accounts dealing with further complications.
} 
But this is a straightforward counterexample to the claim that grounding is irreflexive.

There is, however, something rather unsettling about the counterexample. Diagnosing exactly what has gone astray is not entirely clear. As Krämer notes, it is obviously impredicative in the sense that the propositional quantifier in $\exists p p$ ranges over the proposition it itself helps to express. That is, it ranges over $\exists p p$. If this is to blame, then we would reject the claim that existentially quantified claims are grounded in their instances - they are at best grounded in their predicative instances. That is, we would need to replace (propositional) EXISTENTIAL GROUNDING:

$$
\text { if } A \text { is free for } p \text { in the fact } \varphi(A) \text {, then }\lfloor\varphi(A)<\exists p(\varphi(p))\rfloor
$$

with a restricted principle like PREDICATIVE EXISTENTIAL GROUNDING:

if $A$ is free for $p$ in the predicative fact $\varphi(A)$, then $\lfloor\varphi(A)<\exists p(\varphi(p))\rfloor$

for some more precise explication of predicativity.

It isn't clear how to plausibly implement this strategy; for example, it is hard to see how to formulate a predicativity restriction which is not overly strong. Krämer suggests two ways of doing so: one treats "predicative" in PREDICATIVE EXISTENTIAL GROUNDING as meaning "has no propositional quantifiers", the other postulates a hierarchy of propositions and, thereby, a hierarchy of propositional quantifiers. ${ }^{12}$ Neither way is without obvious problems; more importantly, it is hard to see why impredicativity should be generally worrisome in a grounding context. Our problem case is one where the grounding fact involved quantifies over propositions including itself, but without some additional explanation of why this is problematic, I worry that restricting EXISTENTIAL GROUNDING is a mere palliative remedy. After all, some impredicative grounds sometimes seem fine. Consider 〈every fact is a proposition〉. This, if a fact, is clearly impredicative, but it seems entirely unproblematic to let it ground $\exists p p$, contra PREDICATIVE EXISTENTIAL GROUNDING. It would thus be good to have principled alternatives to Krämer's approach which save the existential grounding principle in full generality even if merely in the service of having theoretical options. ${ }^{13}$

A principled alternative which saves EXISTENTIAL GROUNDING is possible. We can say that EXISTENTIAL GROUNDING is fine, but IRREFLEXIVITY, as stated, is slightly too strong. It is only in the vast majority of cases that something doesn't ground itself; when there is a case of reflexive grounding, this is due to a special feature of the case, the vacuousness of the grounds in grounding the grounded fact. This is not to deny that there are ways of seeing the grounding relation which would motivate trying to solve this puzzle by keeping IRREFLEXIVITY and, perhaps, imposing some requirement of predicativity. If, for example, you thought that grounds were always "more fundamental" than what they ground, or that reality came stratified in

\footnotetext{
${ }^{12}$ See the attempt in [13:? 88] for discussion. There are irritating complications that will arise if you ban propositional quantifiers from quantifying over propositionally quantified formulas. For example, $\forall p(p \rightarrow[(p \wedge s) \vee(p \wedge \neg s)])$ should be a legitimate grounding instance of $\forall r \forall p(p \rightarrow[(p \wedge r) \vee(p \wedge \neg r)])$ by intuitive universal grounding properties - this seems to hold for all propositions $r$ (even and including $s$ itself.) Blocking it seems ad hoc.

${ }^{13}$ For an interesting and attractive alternative response to these puzzles, see [15].
} 
"grounding layers", then it would be natural to ban all reflexive grounding. ${ }^{14}$ After all, it seems nothing can be less fundamental than itself and nothing can be "built" out of itself. ${ }^{15}$ But this is not the only way of understanding the grounding relation; we should have alternative solutions that do not presuppose this particular interpretation of ground.

The irreflexivity of grounding often seems to be motivated by the thought that grounding is explanatory, but that nothing explains itself.

The fact that grounding is systematically connected with explanation in the manner discussed above also suggests that grounding is irreflexive on the assumption that nothing can explain itself. ([31] (p.105) $)^{16}$

A prohibition on reflexive grounding relations seems prima facie reasonable on the assumption that explanation is not reflexive. It, however, is not entirely clear that there are no recherché cases in which something can explain itself in the relevant sense. [16] and [3], among others, have suggested that there are cases of self-explanation; regardless of whether these have to do with grounding in the sense intended here, their suggestions put pressure on the idea that it is obvious that explanation is irreflexive. And, of course, so too do the sort of cases we are discussing.

An anonymous referee asks why the fact $\langle$ there are facts〉 explains that there are facts, instead of just necessitating it. This strikes me as a strange pair of alternatives; that there are facts-i.e. true propositions-is a theorem from my (bog standard) assumptions about propositional quantification. The fact that there are facts is thus trivially necessitated by anything. Many of these necessitations do not bear the least resemblance to anything like an explanation. It seems quite intuitive to me that, in at least a sense, we go further in the case at hand. We seem to explain that there are facts by pointing to an example of one: the very fact that there are facts. If these are not good explanations, it seems to me that it has to be explained why citing any other fact successfully explains that there are some facts, but not the obvious and demonstrable fact that there are some facts. This strikes me as a rather difficult challenge.

At this point, we have put some pressure on the idea that explanation is irreflexive, but not yet on the idea that grounding is irreflexive. However, the support for grounding being irreflexive was supposed to be that explanation is irreflexive. If explanation, in the relevant sense, is sometimes reflexive-and it seems to me that the burden is on the opponent of reflexive explanation to explain away cases or give a compelling argument for irreflexivity-then there seems no initial barrier to considering reflexive cases of explanation. If, therefore, some reflexive explanations seem acceptable,

\footnotetext{
${ }^{14}$ Though see [22] for the claim that it isn't necessary to ban reflexive grounding on the layered picture.

${ }^{15}$ See $\$ 2$ of [3] for the suggestion that, nevertheless, 'God exists' self-grounds. Such cases, similarly to those dealing with ontological dependence, present an alternative challenge to reflexivity and one I am less sympathetic to. Bliss's discussion seems to potentially conflate grounding with related notions like metaphysical causation or ontological dependence, for instance. However, since even metaphysical causation and ontological dependence seem to underwrite explanations, cases like Bliss's present an initial challenge to the putative obviousness of IRREFLEXIVITY as a claim about explanation.

${ }^{16}$ See also [23] and [1] for instances of this move. Lowe [16] claims, in contrast, that self-explanatory states of affairs are possible.
} 
as they do to me, then we should not immediately assume they do not correlate with cases of grounding. ${ }^{17}$

I have here been drawing on intuitions about explanation which are held by at least myself and some others, such as the above cited theorists. I admit that intuitions vary about these cases, but the disagreement seems to me, again, sufficient reason to develop an alternative which preserves the attractive existential grounding principle at what seems to me the minor cost of occasional principled violations of IRREFLEXIVITY.

For those who do not share these intuitions, it is worth noting that there is independent reason to develop alternatives like mine. Some theorists, such as Krämer and Roski [14], have suggested that grounding "backs explanation", but that not all cases of grounding are cases of explanation. ${ }^{18}$ The idea is that grounding is a certain worldly relation which, at least typically, underwrites a relation of explanation between facts, but which is not to be identified with this explanatory relation. This cashes out the sense in which grounding is explanatory without requiring that all features (and, importantly, failures) of explanation imply failures of grounding. On this view, we could maintain that there was something wrong with cases of reflexive grounding - they don't back the best type of metaphysical explanation between facts-but the problem wouldn't be that they weren't instances of grounding.

This is important since, if our main reason for thinking that grounding was irreflexive was that we thought that explanation, in the best sense, was irreflexive, then even if we rejected self-explanation, we might admit that there are cases of reflexive grounding which are not perfectly explanatory. ${ }^{19}$ This would still open up room for a view like mine, since the sort of diagnosis I give of reflexive grounding is easily adaptable, on such a picture, into a claim about explanatory failures of reflexive grounding.

\footnotetext{
${ }^{17}$ It is surprisingly difficult to find an actual argument for the claim that explanation is irreflexive. "Nothing explains itself" is often offered as something like a conceptual truth. However, since some reflexive explanations-like the one I just gave concerning facts and sentences-seem at least prima facie acceptable to some of us, I find this state of affairs rather puzzling. See [11] for related remarks about how to understand IRREFLEXIVITY and worries that our rejection of irreflexivity has to more to do with the pragmatics of offering explanations than with explanations themselves.

${ }^{18}$ See the response to [28], in [14], for discussion and motivation. See also $\$ 3$ of [1], and [12, 29, 30] for useful discussion.

${ }^{19}$ See [14] for numerous detailed examples involving partial grounding. Their idea is that a ground $\gamma$ makes a difference in grounding $\rho$ when, roughly, there is a scenario which contains a full ground of $\rho$ and where subtracting $\gamma$ from that scenario undermines the existence of a total ground in that scenario for $\rho$. They treat scenario as arbitrary collection of facts. This immediately entails, on plausible assumptions, that if a single fact $\gamma$ grounds $\rho$ (and $\rho$ isn't "zero-grounded" in Fine's sense), then $\rho$ is a difference maker. This seems a spandrel of their treatment of scenarios as arbitrary collections of facts; more plausible treatments would allow that some facts are contained in every scenario, such as $\exists p p$. This feature of their account is unfortunate since some cases of reflexive grounding seem to be intuitive cases of (admittedly slightly unusual and different) non-difference-making grounds: $\exists p p$ plausibly is totally grounded in every scenario, but the grounds for it can vary widely and none of the grounds for it is a difference-maker. I hope to compare and integrate their account with mine elsewhere, but this sketch should be enough for the reader to see that they and I are giving voice to similar intuitions. Thanks to a referee for pointing me to their discussion.
} 
In particular, there is an intuitive sense in which cases of vacuous grounding are exactly cases where the grounds are not difference-making grounds in something like Krämer and Roski's sense (see fn. 19 and fn. 24 below.) Insofar as difference-making is a crucial part of explanation, in at least the best sense, there is an explanatory failure in reflexive grounding which doesn't undermine the grounding relations. So it seems that there are a number of pictures of grounding, explanation, and the relationship between them which would underwrite developing a view like mine. ${ }^{20}$

Turning to developing my account, it is important to keep in mind that I do not deny that there is something funny in cases of reflexive grounding. In my view, the important feature of these cases is that the grounds figure in an unusual way in their grounding role. I am not the first to notice this fact; Fine, in a useful discussion of TRANSITIVITY and IRREFLEXIVITY, notes:

...there might be special cases in which $\mathrm{A}$ in one capacity, so to speak, was a ground for $\mathrm{A}$ in another capacity. The truth-teller might in this sense be a ground for itself; and perhaps the cycles involved in our various arguments might also be of this sort. I do not wish to deny that there may be such a notion of ground.

[8] (p. 105)

So even Fine, a proponent of the irreflexivity of ground admits that there might be $a$ notion of ground which admitted of cycles, so long as they are some sort of special case. Fine's thought seems to be that there is more than one way in which a fact might figure in a grounding relation and that reflexive grounding might be okay so as long as these ways don't overlap between the grounds and the grounded fact. ${ }^{21}$ Or, anyways, that for some notions of ground this is true. But we require a story about what these ways might be. ${ }^{22}$

In my view, the difference is that sometimes the content of a fact plays a crucial role in its occurrence as a ground and sometimes it does not. ${ }^{23}$ In particular, sometimes the particular content of a ground is irrelevant to the explanatory work it does in its occurrence as a ground for the grounded fact. How might this occur? Consider the case discussed above of $\langle$ there are some facts $\rangle$. In this case, the content of $\langle$ there are some facts $\rangle$ is important in its occurrence as the grounded fact, but in its occurrence as a ground, its particular content is more or less irrelevant to the work it does as a

\footnotetext{
${ }^{20}$ Thanks to a referee for pushing me to explore this reason for developing my view and suggestions for how this might go.

${ }^{21}$ I make no claim here to Fine exegesis. Read this as "Fine's thought as it struck me". Section 1 of [9] later introduces a notion of weak ground on which every fact weakly grounds itself. This seems a distinct relation from the one he is toying with in the above quotation, where cases of reflexive ground would be special.

${ }^{22}$ Given that Fine and Krämer's puzzles would plausibly arise even for this notion of grounding, if there is such a notion, then we need a story for it even if the solution for some other target notion of grounding is to bar impredicative instances of EXISTENTIAL GROUNDING.

${ }^{23} \mathrm{An}$ anonymous referee suggests that there is something funny with talking about the occurrence of a fact as a ground. Since I take facts to be true propositions, grounding to be a relation between propositions, and grounding claims to be propositions about grounding relations, I see nothing funny about this, but if it bothers the reader, take my use of 'occurrence' to be a vulgar way of indicating the occurrence of a sentence-like entity, denoting a true proposition, in a claim about grounding; a claim that, if true, corresponds to a real grounding relation.
} 
ground. Any fact would have done in its stead. So, intuitively, there is nothing in particular about what it says that is important for it grounding that there are some facts. We could as easily explain that there are facts by pointing to the fact that there are monkeys, the fact that I am wearing red sneakers, or the fact that one is the loneliest number. $^{24}$

To put the point slightly differently, it seems to me that one intuition behind the claim that grounding is irreflexive is that it is a mystery how the content of some fact could explain the content of that very fact. However, the particular content of $\exists p p$ doesn't seem to matter to the role that it plays in explaining $\exists p p$. It does matter to the role that $\exists p p$ plays in being grounded by $\exists p p$ or any other fact. So Fine and Krämer's examples seem, on closer inspection, to display at least one way that a fact might figure in a grounding relation in two different ways.

In many cases, the particular content matters quite a bit. The particular content of the fact that I am wearing red sneakers matters in grounding the fact that I am wearing shoes of some color. And, again, in our case of reflexive grounding, the particular content matters to the grounded fact-since if the content were different in certain ways, then it would not be grounded by itself. 〈there are some interesting facts〉 is not grounded by itself, after all. If we can capture this difference slightly more precisely, we can put some flesh on Fine's important point about two different roles for facts in grounding claims.

The key move in giving a precise account of the difference is distinguishing between vacuous and non-vacuous grounds. ${ }^{25}$ The notion of a vacuous ground is modeled on Quine's notion of a vacuous occurrence: for Quine, an expression $\varphi$ occurs vacuously in the true sentence $\varphi \vee \neg \varphi$ since we could substitute any grammatically appropriate expression $\psi$ for $\varphi$ in $\varphi \vee \neg \varphi$ without disturbing its truth. Quine took this to show that (the content of) $\varphi$ plays no essential role establishing the truth of $\varphi \vee \neg \varphi$ [20]. I will shortly propose that in any plausible case of reflexive grounding, the grounded fact occurs vacuously, in an analogous sense, in the grounds. Note, "propose", not "prove". What I show is that Fine and Krämer's cases can be dealt with in this way and that the solution is natural. Again, I do not show that there are no other worrisome cases of irreflexive grounds; I view Fine and Krämer's casesas they are constructed out of logical and "logic of grounding"-ical materials-as the most serious challenge to IRREFLEXIVITY. ${ }^{26}$

\footnotetext{
${ }^{24}$ This is the sense of some reflexive grounds not being difference-makers I pointed at above. Since the particular content of the grounds doesn't matter here, they are intuitively not difference-making grounds. Any old fact, with any old content, would do equally well; moreover, in this particular case, since there are always some facts, we will always have a total ground for $\langle$ there are some facts $\rangle$. Again, I hope to explore in detail the analogy to Krämer and Roski's account on another occasion.

${ }^{25}$ This solution first occurred to me while thinking through Charles Pigden's account of ethical autonomy in terms of Quine's notion of vacuity [18]. The notion of vacuity used here is different, of course, since the context is explanation, not entailment.

${ }^{26}$ Other potential cases beyond those so far mentioned, such as those in [11] and [25], involve significant further complications involving identity and truth, and again, potentially admit of independent resolution. Fine and Krämer's cases arise from basic facts about logic and the logic of grounding and are thus significantly more threatening. If there needs to be further work done to salvage the irreflexive property from these additional sort of worries, well and good. But that doesn't show that my proposal doesn't capture exactly what has gone wrong with the Fine and Krämer puzzles, even in contexts where further challenges may arise.
} 
Quine's notion of vacuous occurrence largely captures the sense in which the content of a particular sentence can be irrelevant to the truth of what it entails or of a complex sentence it occurs within. We want to do something similar with respect to content and metaphysical explanation. We want to define a notion of vacuity which captures when the particular content of grounds is inessential to the metaphysical explanation of why the grounded fact holds. The idea is simple. Consider a grounding claim $\lfloor A<C\rfloor$ and structurally identical grounding claim $\lfloor B<C\rfloor$ we obtain by (uniformly) substituting an arbitrary proposition $B$ for $A$. Suppose this new grounding claim holds at any world where $B$ holds. What does this show us about $A$ ? It shows that the particular content of $A$ was inessential to the grounding explanation as any proposition, at any world where it is true, would have done in its place. ${ }^{27}$

This does not mean that we could simply delete $A$ from the grounds $-A$ plays an explanatory role in the actual explanation of $C$ - but it does mean that $A$ 's particular content is not relevant since any content would do in its stead. ${ }^{28}$ Note the essential occurrence of 'particular' in the above discussion. I am not claiming that the content does not matter in the actual explanation. That a particular proposition has some content and that that content is true or false will nearly always matter in providing a grounding explanation. But the particular content will sometimes not matter; that is, it will not matter what the content of the proposition is, merely that it has content and that that content is true or false (see objection 8 below.)

More formally, given a (total) grounding fact $\gamma$, let $\gamma^{A / B}$ be the result of uniformly substituting the proposition $B$ for $A$ in the grounds of $\gamma$. Suppose $\gamma$ is $\lfloor A, A \vee C<$ $G\rfloor \cdot \gamma^{A / B}$ is then $\lfloor B, B \vee C<G\rfloor \cdot \gamma^{A / B}$ might not be a grounding fact since B might be false. Note that $B$ might occur unembedded in $\gamma$ or it might occur in $\gamma$ by being embedded in some more complex proposition. We will say that:

A proposition $A$ occurs vacuously in a grounding fact $\gamma$ when $A$ occurs in the grounds of $\gamma$ and, for any proposition $B, \gamma^{A / B}$ holds anywhere the (modified) grounds of $\gamma^{A / B}$ are true. ${ }^{29}$

The proposition $\exists p p$ is vacuous in $\lfloor\exists p p<\exists p p\rfloor$ since substituting any proposition $A$ for $\exists p p$ in the grounds results in a true grounding claim anywhere $A$ holds.

\footnotetext{
${ }^{27}$ It is important to "modalize" here since otherwise, we might get that we can freely substitute facts for $A$, but only because there is an insufficient slate of available facts to put in the place of $A$. In such a case, $A$ wouldn't be truly vacuous. Of course, my definition of vacuity is less permissive than the definition just mentioned, so my use of vacuity shouldn't trouble someone tempted by the weaker definition.

${ }^{28}$ Note that vacuity is meant to be a sufficient condition for the particular content of a fact playing no essential role in a ground relation. It may not be a necessary condition, though it strikes me as plausible that it is. I hope to explore this issue elsewhere.

${ }^{29}$ The grounded fact might be such that it holds in no world other than the actual world. Say, for example, the grounded fact $C$ is a rigidified fact holding only in the actual world. For full generality, we would want to work with a more qualitative notion of the identity of propositions and say, strictly speaking, that $A$ is B-vacuous in $\lfloor A<C\rfloor$ when $\lfloor B<C *\rfloor$ for $C *$ the qualitative analogue of $C$ in the world in which $B$ is true. See [33] for more details. For the examples I discuss here, nothing turns on this point. Also, for a truly adequate definition, we would have to deal with complications involving simultaneous substitutions, as Quine later pointed out [21] (fn. 2). Doing this is slightly more difficult in a grounding context; I demonstrate one way of doing so, involving making explicit various property connections, in [33]. For the cases under consideration, this isn't really necessary, so I will not discuss the matter is more detail here.
} 
Note that we have performed the substitution only in the grounds, not in the grounded fact. If we substituted everywhere in the grounding fact, then many constituents of many grounding claims - such as any $A$ in logical relationships like $\lfloor A<A \vee B\rfloor$ would turn out to be vacuous. This is not the phenomenon we are attempting to capture; instead, we want to capture how the particular content of the grounds might not be relevant for its work in grounding some particular. In the case of $\lfloor A<A \vee B\rfloor$, the content of $A$ matters quite a bit for its role in grounding $A \vee B$. Not any old content would do. ${ }^{30}$

In the cases under consideration, what is important is only that $\exists p p$ is fact. That's all that is needed for it to ground $\exists p p$. Of course, the content of $\exists p p$ is, in a way, part of the content of $\exists p p$. $\exists p$ ranges over all propositions and since $\exists p p$, being a fact, is a true proposition, it's in the range of $\exists p$. But not all cases of vacuous grounding are cases where the content of the grounded fact includes the content of the grounds.

Consider an illustrative example: that Yağmur answered my question correctly might be grounded in the fact that Yağmur answered with $A$, that $A$ is about rain, $A$, and that I asked Yağmur for an example of a fact about rain. If we uniformly substitute $B$ for $A$ in the grounds, then we get that Yağmur answered with $B, B$, that $B$ is about rain, and that I asked Yağmur for an example of a fact about rain. This plausibly grounds that Yağmur answered my question correctly and would do so no matter what $B$ was, in any case where $B$ is about rain, where $B$ held, and where the other modified grounds hold. That is, in any case where the modified grounds are true, they ground that Yağmur answered my question correctly. So $A$ is vacuous in this grounding fact. The particular content of $A$, just as in the reflexive cases which are our main concern, do no work in explaining why Yağmur answered my question correctly-since all that matters is that $A$ is about rain (which is itself part of the grounds.) See also objection and reply 8 below for more discussion. ${ }^{31}$

With the notion of vacuous grounds now spelled out, I propose SUBSTANTIVE IRREFLEXIVITY:

$$
\lfloor\ldots, A \nless A\rfloor \text { unless } A \text { occurs vacuously in }\lfloor\ldots, A<A\rfloor
$$

as the proper articulation of the irreflexivity of grounding. Given SUBSTANTIVE IRREFLEXIVITY, we can retain the full-blooded form of EXISTENTIAL GROUNDING without relinquishing the core intuition which drove (at least some of) us to accept the irreflexivity of grounding. We merely need to accept that sometimes-as in our existential case-we can have vacuous reflexive grounds.

Moreover, this proposal makes sense of Fine's observation that facts can occur in two capacities in grounding claims. When the capacities are different in the way suggested above-when the particular content of a fact matters to its occurrence as the grounded fact, but not to its occurrence in the grounding facts-we can allow reflexive grounding while preserving the intuition that the particular content of a fact

\footnotetext{
${ }^{30}$ This is rather important. If we substituted throughout the grounding claim, vacuity would be everywhere. Intuitively though, vacuity, at least vacuity of entire propositions in a grounding fact, is a fairly rare phenomena. See [33] for a more detailed definition of vacuity which covers parts of propositions as well.

${ }^{31}$ For significantly more discussion of these sorts of cases, especially for cases involving the relationship of normative and natural facts such as promises, see [33].
} 
doesn't metaphysically explain its particular content. This because it is only when the particular content of $A$ doesn't matter-when $A$ is vacuous in $\lfloor\ldots, A<A\rfloor-$ that $A$ can ground itself. Since my suggestion preserves this essential intuition about metaphysical explanation without abandoning the extremely plausible EXISTENTIAL GROUNDING principle, SUBSTANTIVE IRREFLEXIVITY is a serious contender for a way of resolving for Fine and Krämer's puzzles.

\section{Objections and Replies}

Objection 1 Let $\operatorname{Pos}(A)$ hold of a proposition $A$ if and only if $A$ contains no negations. Intuitively, that there are any positive propositions at all is grounded by, among other things, that there are some positive propositions; after all, the latter is a positive proposition. But, since $\exists p \operatorname{Pos}(p)$ would not be vacuous in

$$
\lfloor\exists p \operatorname{Pos}(p)<\exists p \operatorname{Pos}(p)\rfloor
$$

we face the question of whether we can run an analogue of Krämer's argument which has this result.

Reply No. Suppose we have $\exists p \operatorname{Pos}(p)$. By generalizing, we get that $\lfloor\exists p \operatorname{Pos}(p)<$ $\exists p p\rfloor$, but we have no way to generalize so as to directly get $\lfloor\exists p \operatorname{Pos}(p)<$ $\exists p \operatorname{Pos}(p)\rfloor$.

Objection 2 Suppose we had a further superficially plausible principle of total grounding for some property like positivity:

$$
\text { Positive grounding: if } A \text { and } \operatorname{Pos}(A) \text {, then }\lfloor A<\operatorname{Pos}(A)\rfloor
$$

So long as the property of being positive can itself apply to a propositionally quantified existential fact like $\exists p \operatorname{Pos}(p)$, POSITIVE GROUNDING and EXISTENTIAL GROUNDING together with the transitivity of grounding will yield a problem so long as, as seems immune from doubt, there are some true positive propositions:

1. $\exists p \operatorname{Pos}(p)$ and $\operatorname{Pos}(\langle\exists p \operatorname{Pos}(p)\rangle)$

2. $\lfloor\exists \operatorname{PPos}(p)<\operatorname{Pos}(\langle\exists p \operatorname{Pos}(p)\rangle)\rfloor$

(POSITIVE GROUNDING)

3. $\lfloor\operatorname{Pos}(\langle\exists p \operatorname{Pos}(p)\rangle)<\exists p \operatorname{Pos}(p)\rfloor$

(EXISTENTIAL GROUNDING)

4. $\lfloor\exists p \operatorname{Pos}(p)<\exists p \operatorname{Pos}(p)\rfloor$

(TRANSITIVITY)

But (4) violates SUBSTANTIVE IRREFLEXIVITY since $\exists p \operatorname{Pos}(p)$ is not vacuous in $\lfloor\exists p \operatorname{Pos}(p)<\exists p \operatorname{Pos}(p)\rfloor$.

Reply We cannot retain all of POSITIVE GROUNDING, EXISTENTIAL GROUNDING, and SUBSTANTIVE IRREFLEXIVITY so long as there are some true positive propositions. Happily, POSITIVE GROUNDING, it turns out, is entirely implausible on closer inspection.

It is not plausible that the fact $A$ explains the positivity of $A$. What seems to do the explaining here is the fact that $A$ is the way that it is; that is, that $A$ has the structure that it has. But $A$ and 〈that $A$ is the way that it is〉 are distinct facts. There are, as 
a result, two distinct general grounding claims here. POSITIVE GROUNDING, where $\operatorname{Pos}(A)$ is totally grounded in $A$ and POSITIVE STRUCTURE GROUNDING:

$$
\text { if } \operatorname{Pos}(A),\lfloor A \text { is the way it is }<\operatorname{Pos}(A)\rfloor
$$

POSITIVE STRUCTURE GROUNDING is a very plausible grounding claim, POSITIVE GROUNDING not. Whatever initial plausibility POSITIVE GROUNDING has seems to me to drain away once we see POSITIVE STRUCTURE GROUNDING. But, if this does not yet convince, consider the fact that grounding is widely considered to be "factive". That is, when we have a true grounding claim $\lfloor A<B\rfloor$, then $A$ and $B$ must also be true (i.e. they must be facts, not mere propositions.) But it seems clear that $A$ figures, in some way, in the explanation of the fact that $A$ is positive, regardless of whether or not $A$ is true. So it must be that whatever the actual ground of $A$ 's positivity is, it's not the proposition $A$ itself, but something closer to the proposition that $A$ is the way it is which is true even if $A$ is not. ${ }^{32}$

But if POSITIVE STRUCTURE GROUNDING, not POSITIVE GROUNDING, is the correct way to see the relation between, say, $A$ and $A$ 's positivity, then there is no immediate threat arising for my solution. And, clearly, the same goes for properties like [is a quantified proposition], [contains purple as a constituent], or what have you. So it is not at all obvious that properties like positivity that will cause us a problem since it is not at all clear that there are properties $F$ such that there are some $F$ propositions, $\lfloor\exists p F(p) \nless \exists p F(p)\rfloor$, and where the F-analogue of POSITIVE GROUNDING holds. ${ }^{33}$

Objection 3 Let $F$ be the open formula $p \vee A$ where $p$ is a free propositional variable. Since

$$
\text { Disjunctive Grounding : if } A \text {, then }\lfloor A<A \vee B\rfloor
$$

holds and since $\exists p[(p \vee A)$ grounds $\exists p(p \vee A) \vee A)]$, we can use the above style of argument to conclude that $\lfloor\exists p(p \vee A)<\exists p(p \vee A)\rfloor$. So isn't there still a problem here?

Reply Since not only $\lfloor\exists p(p \vee A)<\exists p(p \vee A)\rfloor$, but also $\lfloor B<\exists p(p \vee A)\rfloor$ for any proposition $B, \exists p(p \vee A)$ occurs vacuously in $\lfloor\exists p(p \vee A)<\exists p(p \vee A)\rfloor$ and there is no conflict with SUBSTANTIVE IRREFLEXIVITY.

Objection 4 Your solution is merely a solution to the analogue of Krämer's puzzle for total grounding. As Krämer (and Fine's) puzzles are developed for partial grounding, your solution is incomplete.

\footnotetext{
${ }^{32}$ See [31] for a discussion of the claim that objects don't ground anything. Rather, their existence or properties do the grounding. The point is attributed to Shamik Dasgupta.

${ }^{33}$ Note that even if we accept notions of "object" grounding"- grounding $F(A)$ in the proposition $A$ itself considered as an object—-there is still no threat to our argument. We cannot infer $\lfloor\exists p F(p)<\exists p F(p)\rfloor$ from $\lfloor\exists p F(p) \stackrel{o}{<} F(\langle\exists p F(p)\rangle)\rfloor$ and $\lfloor F(\langle\exists p F(p)\rangle)<\exists p F(p)\rfloor$ since $<$ and $\stackrel{o}{<}$ are distinct relations. And there is no reason to think that if $o$ object-grounds $A$ and $A$ grounds $B$, that $o$ grounds $B$. At best, $o$ object grounds $B$, but this is not violation of irreflexivity. See [27] for motivation of the idea that objects can stand in grounding relations.
} 
Reply Yes, as stated, but the solution extends naturally to a solution to puzzles involving partial grounding. ${ }^{34}$ It is worth spelling this out a bit since we have cases of reflexive partial grounding which hold where their total grounding analogues fail. For example, using $\prec$ for partial grounding — being part of a set of total grounds - and Pos Fac for the property of being a positive fact (i.e. a true positive proposition)—it seems to me that

$$
\lfloor\exists p \operatorname{Pos} F a c(p) \prec \exists p \operatorname{Pos} F a c(p)\rfloor
$$

but that the total ground analogue

$$
\lfloor\exists \operatorname{PPos} F a c(p)<\exists p \operatorname{Pos} F a c(p)\rfloor
$$

fails. I argue for this by drawing on my response to objection 3 and the assumption that facts are true propositions. If we consider facts to be true propositions, then it would be strange to hold that we ground $\exists p \operatorname{Pos}(p)$ in $\operatorname{Pos}(\langle\exists p \operatorname{Pos}(p)\rangle)$, but we don't analogously need PosFac $(\langle\exists p \operatorname{Pos} F a c(p)\rangle)$ among the grounds of $\exists p \operatorname{Pos} F a c(p)$. It is much more natural to hold that we can ground $\exists p \operatorname{Pos} F a c(p)$ by the fact that it holds and the fact that it is positive. So, the proper grounding fact here is:

$$
\lfloor\exists p \operatorname{Pos} F a c(p), \operatorname{Pos} F a c(\langle\exists p \operatorname{Pos} F a c(p)\rangle)<\exists p \operatorname{Pos} F a c(p)\rfloor
$$

since we need both that $\exists \operatorname{pPos} F a c(p)$ holds and that it is positive in order to ground that there are positive facts. This grounding fact is unproblematic since $\exists \operatorname{POSFac}(p)$ occurs vacuously in

$$
\lfloor\exists p \operatorname{Pos} F a c(p), \operatorname{Pos} F a c(\langle\exists p \operatorname{Pos} F a c(p)\rangle)<\exists p \operatorname{Pos} F a c(p)\rfloor
$$

since for any other proposition $B$ :

$$
\lfloor B, \operatorname{Pos} F a c(B)<\exists p \operatorname{Pos} F a c(p)\rfloor
$$

holds whenever $B$ and $\operatorname{Pos} F a c(B)$ do. ${ }^{35}$ Now, so long as we define vacuous occurrence for partial grounding in terms of a component occurring vacuously in any set of total grounds extending the partial grounds-as seems entirely natural given what partial grounding is-reflexive partial grounds will cause us no significant worry. We can advocate, that is, PARTIAL SUBSTANTIVE IRREFLEXIVITY:

$\lfloor\ldots A \ldots \nprec A\rfloor$ unless $A$ occurs vacuously in $\lfloor\ldots A, \Gamma \ldots<A\rfloor$ for all $\Gamma$ such that $\lfloor\ldots A, \Gamma, \ldots<A\rfloor$.

That is, if for every way of cashing out $\Gamma$ which produces a legitimate grounding fact, substituting $B$ for $A$ uniformly also produces a grounding fact when we assume the modified grounds hold. The explanation of PARTIAL SUBSTANTIVE IRREFLEXIVITY is entirely analogous to the the explanation of SUBSTANTIVE IRREFLEXIVITY, viz. when $A$ is vacuous in a grounding fact, then its content is not relevant to the work it does in grounding the grounded fact.

\footnotetext{
${ }^{34}$ Though this is obvious, the definition of vacuity smoothly adapts to the case of partial grounding.

${ }^{35}$ We are replacing $\exists p \operatorname{Pos} F a c(p)$ in both sentential and argument position. This is unproblematic since grounding relates propositions which can have as part of their structure other propositions.
} 
Objection 5 What about cases of reflexive universal grounding?

Reply The solution given above can be shown to easily solve reflexive cases of universal grounding as well. Consider, for example, the claim that:

$$
\text { ŁTOTALity } \left., A_{0}, A_{1}, \ldots<\forall p p \vee \neg p\right\rfloor
$$

where the $A_{n}$ s are all the facts and TOTALITY is the "totality fact" testifying to this. ${ }^{36}$ If we uniformly substitute some proposition $B$ for some proposition $A_{n}$ in the grounds of the above indented grounding claim, do we end up with a case in which all the grounds could be true, but they don't ground $\forall p p \vee \neg p$ ? No, since we have to uniformly substitute. Since $A$ will occur in TOTALITY, on reasonable treatments of TOTALity, we need to also substitute $B$ for $A$ in TOTALITY and this will return a different totality fact TOTALITY ${ }^{A_{n} / B}$. Presumably, though,

$$
\left\lfloor\text { TOTALity }^{A_{n} / B}, A_{0}^{A_{n} / B}, A_{1}^{A_{n} / B}, \ldots B \ldots<\forall p p \vee \neg p\right\rfloor
$$

in any case in which TOTALITY ${ }^{A_{n} / B}, A_{0}^{A_{n} / B}, A_{1}^{A_{n} / B}, \ldots B$ are true. ${ }^{37}$

Objection 6 Consider a paradox of knowledge (from [10]) stemming from two principles:

- An existential generalization is totally grounded by each of its true instances.

- For any $A$, if Obama knows $A$, then this is partly grounded by $A$.

Writing "Obama knows" as $O_{k}$, we get from the first that $\left\lfloor O_{k}\left(\exists p O_{k}(p)\right)<\right.$ $\left.\exists p O_{k}(p)\right\rfloor$ and from the second that $\left\lfloor\exists p O_{k}(p) \prec O_{k}\left(\exists p O_{k}(p)\right)\right\rfloor$. By transitivity for partial grounding, we get $\left\lfloor O_{k}\left(\exists p O_{k}(p)\right) \prec O_{k}\left(\exists p O_{k}(p)\right)\right\rfloor$ but $O_{k}\left(\exists p O_{k}(p)\right)$ is not vacuous in this grounding fact. ${ }^{38}$

\footnotetext{
${ }^{36}$ I make no assumptions we can index all the propositions here. I'm using the ' $n$ ' notation simply to make exposition easier.

${ }^{37}$ This solution assumes that in the proper account of universal grounding, the TOTALITY fact appears in the grounds. This can be avoided; I use the technical notion of grounding* facts in [33] to avoid taking a stand on which grounds are necessary for a grounding claim to go through. This is related, though not identical to, the distinction between grounds and enablers; roughly, grounding* facts are grounding facts "fleshed-out" with facts which are not part of the explanation, but which are necessary in order to make sense of the meaning and role of the facts in the explanation so as to allow the proper substitutions to go through. This includes enablers (see [4] for discussion of enablers) and certain privileged property connections. For the point I'm making here, such technical machinations aren't to the point. The interested reader can consult [33] for discussion.

${ }^{38}$ Thanks to a helpful referee for suggesting I consider this case. The cognoscenti might here wonder about the truth-teller and how I deal with it. It is a complicated case that I hope to deal with elsewhere. Roughly, the trouble is that the mechanism by which self-reference is achieved can involve significant background materials which are obfuscated by writing things like "let $\varphi$ be $\varphi$ is true". (This is a general problem. See [5] for a useful discussion of this mistake in the literature on paradoxes of validity.) Depending on how we make sense of self-reference, the solution will vary as will the plausibility of the claim that the truthteller grounds itself. As for the general plausibility of the claim that the truth-teller grounds itself, note how easily our intuition slips from "obviously it grounds itself", when we write the grounding claim of a truth-teller $\gamma$ as $\lfloor\gamma<\gamma$ is true $\rfloor$ to "clearly it doesn't ground itself" when we write it as $\lfloor\gamma$ is true $<\gamma\rfloor$. Caution here is warranted.
} 
Reply In order to resolve this puzzle, we need to probe a bit deeper into how the puzzle arises. The important move is replacing $\exists p O_{k}(p)$ with $O_{k}\left(\exists p O_{k}(p)\right)$ to get the case of reflexive partial grounding. Partial grounds need fleshing out though. Consider replacing $\left\lfloor O_{k}\left(\exists p O_{k}(p)\right)<\exists p O_{k}(p)\right\rfloor$ with $\left\lfloor O_{k}(B)<\exists p O_{k}(p)\right\rfloor$. We can then obtain, in the same way, $\left\lfloor O_{k}(B) \prec O_{k}\left(\exists p O_{k}(p)\right)\right\rfloor$. This is an extremely partial grounding fact, suggesting we need to flesh it out a lot more in order to assess the total grounding fact. What we need, intuitively, is a ground expressing the relationship of Obama's knowledge that $B$ to the grounded fact and, more importantly, Obama's knowledge about this relationship. Without this last, it is entirely unclear how to get a plausible total grounding fact from the partial grounding fact.

To make this clearer, let's actually flesh out the partial grounding fact $\left\lfloor O_{k}(B) \prec\right.$ $\left.O_{k}\left(\exists p O_{k}(p)\right)\right\rfloor$ to a plausible total grounding fact. It would have to be something like:

$$
\left\lfloor O_{k}(B), O_{j b}\left(O_{k}(B)\right), O_{k}\left[O_{k}(B) \rightarrow \exists p O_{k}(p)\right]<O_{k}\left(\exists p O_{k}(p)\right)\right\rfloor
$$

where $O_{j b}(A)$ represents that Obama is justified in believing that $A .^{39}$ If we similarly expand $\left\lfloor O_{k}\left(\exists p O_{k}(p)\right) \prec O_{k}\left(\exists p O_{k}(p)\right)\right\rfloor$, writing $B$ in the grounds for $O_{k}\left(\exists p O_{k}(p)\right)$ for both typesetting and sneaky reasons, we get:

$$
\left.\left\lfloor B, O_{j b}(B), O_{k}\left[B \rightarrow \exists p O_{k}(p)\right)\right]<O_{k}\left(\exists p O_{k}(p)\right)\right\rfloor
$$

But $O_{k}\left(\exists p O_{k}(p)\right)$ is vacuous in this grounding fact. Letting $B$ now serve as an arbitrary proposition, so the above is the result of substituting $B$ for $O_{k}\left(\exists p O_{k}(p)\right)$ in the grounds, the above is a grounding fact anywhere the modified grounds are true. So the partial grounding fact $\left\lfloor O_{k}\left(\exists p O_{k}(p)\right) \prec O_{k}\left(\exists p O_{k}(p)\right)\right\rfloor$ doesn't violate PARTIAL SUBSTANTIVE IRREFLEXIVITY (see objection 5). ${ }^{40}$

Objection 7 The intuitive gloss you have given and motivated of the content not mattering comes apart from the formal gloss in terms of a vacuous occurrence. Consider, for example, the fact that $\lfloor$ Barry believes that snow is white, that snow is white concerns snow $<$ Barry believes something concerning snow $\rfloor$. 〈snow is white $\rangle$ occurs vacuously in this grounding fact-for arbitrary $A, \mathrm{~L}$ Barry believes $A, A$ concerns snow $<$ Barry believes something concerning snow $\rfloor$-but intuitively, that snow is white is not irrelevant to explaining the fact that Barry believes something concerning snow. ${ }^{41}$

Reply I deny the counterexample is a counterexample. It is exactly the right result that that snow is white occurs vacuously in this grounding fact; the particular content

\footnotetext{
${ }^{39}$ Or whatever you take the additional materials grounding the fact that Obama knows something to be.

${ }^{40}$ Readers might wonder here about concerns involving the opacity of knowledge claims. Careful inspection of my tentative solution to this puzzle reveals that I have made no assumptions about opacity here, though a more thorough survey and defense of my expansion claim might. The interesting issue of the relationship of substitution into grounding contexts can be bracketed here given that it is generally orthogonal to my concerns.

${ }^{41}$ Thanks to Jon Litland and a helpful referee for raising cases of this form. Similar responses hold for the variations on this case suggested by the referee, so I will just use the simple version they first suggest.
} 
of $\langle$ snow is white $\rangle$ plays no role in grounding that Barry believes something concerning snow. What plays the important role in grounding the grounded fact is the fact that $\langle$ snow is white concerns snow, which is explicitly part of the grounds. If this fact is needed for the grounding claim, then we have no counterexample here since what does the work in explaining that Barry believes something concerning snow is that the proposition he believes concerns snow. Of course, if we replaced that snow is white with that grass is green, we would have as one of the grounds that /grass is green $\rangle$ concerns snow, which is manifestly false, but if it were true, we would have a perfectly fine explanation.

To see the point here, contrast this case with another putative grounding claim: $L$ Barry believes that snow is white $<$ Barry believes something concerning snow $\rfloor$. If this is a correct grounding claim-i.e. if we do not need 〈snow is white〉 concerns snow in our explanation - then it would indeed be problematic if 〈snow is white〉 were vacuous in this contracted grounding claim. But, it's not. That Barry believes that grass is green does not ground that Barry believes something concerning snow.

This shows that it really is the fact that 〈snow is white) concerns snow that is doing the actual explanatory work in the grounding claim in the objection. The particular content of 〈snow is white $\rangle$ doesn't play a role in the putatively problematic example, except as articulated as part of the grounds (i.e. in its occurrence as the fact snow is white) concerns snow.) Of course, which of these two grounding claims correctly articulates the grounds of Barry's snow-concerning believing is a matter of some contention, but the important fact is that neither constitutes a problem for my analysis.

Acknowledgments Thanks to Barry Maguire, Shamik Dasgupta, Catharine Diehl, David Kovacs, Jon Litland, Beau Madison Mount, Mike Raven, Gideon Rosen, and a couple of helpful referees for very useful discussion.

Open Access This article is distributed under the terms of the Creative Commons Attribution 4.0 International License (http://creativecommons.org/licenses/by/4.0/), which permits unrestricted use, distribution, and reproduction in any medium, provided you give appropriate credit to the original author(s) and the source, provide a link to the Creative Commons license, and indicate if changes were made.

\section{References}

1. Audi, P. (2012). A clarification and defense of the notion of grounding. In Correria, R., \& Schnieder, B. (Eds.) Metaphysical Grounding: Understand the Structure of Reality (pp. 101-121): Cambridge University Press.

2. Barnes, E. (forthcoming). Symmetric dependence. In Bliss, R., \& Priest, G. (Eds.) Reality and its Structure: Oxford University Press.

3. Bliss, R. (forthcoming). Grounding and reflexivity. In Bliss, R., \& Priest, G. (Eds.) Reality and its Structure: Oxford University Press.

4. Bliss, R., \& Trogdon, K. (2016). Metaphysical Grounding. In Zalta, E.N. (Ed.) The Stanford Encyclopedia of Philosophy: Spring 2016 edn.

5. Cook, R.T. (2014). There is no paradox of logical validity. Logica Universalis, 8(3-4), 447-467.

6. Donaldson, T. (forthcoming). The (metaphysical) foundations of arithmetic? Noûs.

7. Fine, K. (2001). The question of realism. Philosophers' Imprint, 1(1), 1-30.

8. Fine, K. (2010). Some puzzles of ground. Notre Dame Journal of Formal Logic, 51(1), 97-118.

9. Fine, K. (2012). The pure logic of ground. Review of Symbolic Logic, 5(1), 1-25. 
10. Howard-Snyder, D., Rasmussen, J., \& Cullison, A. (2013). On Whitcomb's grounding argument for atheism. Faith and Philosophy, 30(2), 198-204.

11. Jenkins, C.S. (2011). Is metaphysical dependence irreflexive? The Monist, 94(2), 267-276.

12. Kovacs, D.M. Grounding and the argument from explanatoriness. Philosophical Studies (forthcoming).

13. Krämer, S. (2013). A simpler puzzle of ground. Thought: A Journal of Philosophy, 2(2), 85-89.

14. Krämer, S., \& Roski, S. Difference-making grounds. Philosophical Studies (forthcoming).

15. Litland, J.E. (2015). Grounding, explanation, and the limit of internality. Philosophical Review, 124(4), 481-532.

16. Lowe, E.J. (1998). The possibility of metaphysics: Substance, identity, and time: Oxford University Press.

17. Paseau, A. (2010). Defining ultimate ontological basis and the fundamental layer. Philosophical Quarterly, 60(238), 169-175.

18. Pigden, C.R. (1989). Logic and the autonomy of ethics. Australasian Journal of Philosophy, 67(2), 127-151.

19. Prior, A.N. (1971). Objects of Thought. Oxford University Press.

20. Quine, W.V. (1936). Truth by convention. Journal of Symbolic Logic, 1, 77-106.

21. Quine, W.V. (1960). Carnap and logical truth. Synthese, 12(4), 350-374.

22. Rabin, G.O. (forthcoming). Grounding orthodoxy and the layered conception. In Bliss, R., \& Priest, G. (Eds.) Reality and its Structure: Oxford University Press.

23. Raven, M.J. (2012). In defence of ground. Australasian Journal of Philosophy, 90(4), 687-701.

24. Raven, M.J. (2015). Ground. Philosophy Compass, 10(5), 322-333.

25. Rodriguez-Pereya, G. (2015). Grounding is not a strict order. Journal of the American Philosophical Association, 1, 517-534.

26. Rosen, G. (2010). Metaphysical dependence: Grounding and reduction. In Hale, B., \& Hoffmann, A. (Eds.) Modality: Metaphysics, Logic, and Epistemology (pp. 109-36): Oxford University Press.

27. Schaffer, J. (2009). On what grounds what. In Manley, D., Chalmers, D.J., \& Wasserman, R. (Eds.) Metametaphysics: New Essays on the Foundations of Ontology (pp. 347-383): Oxford University Press.

28. Schaffer, J. (2012). Grounding, transitivity, and contrastivity. In Correia, F., \& Schnieder, B. (Eds.) Metaphysical Grounding: Understanding the Structure of Reality (pp. 122-138): Cambridge University Press.

29. Schaffer, J. (2016). Grounding in the image of causation. Philosophical Studies, 173(1), 49-100.

30. Schnieder, B. (2015). The asymmetry of 'because'. Grazer Philosophische Studien, 9, 131-164.

31. Trogdon, K. (2013). An introduction to grounding. In Hoeltje, M., Schnieder, B., \& Steinberg, A. (Eds.) Varieties of Dependence: Ontological Dependence, Grounding, Supervenience, ResponseDependence (Basic Philosophical Concepts) (pp. 97-122): Philosophia Verlag.

32. Williamson, T. (2013). Modal Logic as Metaphysics. Oxford University Press.

33. Woods, J. On vacuous grounding: The case study of ethical autonomy (manuscript). 